\title{
Genetic and Environmental Influences on Individual Differences in Sleep Duration During Adolescence
}

\author{
Saskia J. te Velde, 1,2,* Niels van der Aa, ${ }^{6, *}$ Dorret I. Boomsma, ${ }^{1,3}$ Eus J. W. van Someren, ${ }^{4,5}$ \\ Eco J. C. de Geus, ${ }^{1,3}$ Johannes Brug, ${ }^{1,2}$ and Meike Bartels ${ }^{1,3}$ \\ ${ }^{1} \mathrm{EMGO}^{+}$Institute for Health and Care Research, VU University Medical Centre, Amsterdam, The Netherlands \\ ${ }^{2}$ Department of Epidemiology and Biostatistics, VU University Medical Centre, Amsterdam, The Netherlands \\ ${ }^{3}$ Department of Biological Psychology, VU University, Amsterdam, The Netherlands \\ ${ }^{4}$ Netherlands Institute for Neuroscience (NIN), Royal Netherlands Academy of Arts and Sciences, Amsterdam, The \\ Netherlands \\ ${ }^{5}$ Department of Integrative Neurophysiology, Neuroscience Campus Amsterdam, VU University, Amsterdam, The \\ Netherlands \\ ${ }^{6}$ Stichting Centrum '45, Oegstgeest, The Netherlands
}

\begin{abstract}
This study assessed to what extent genetic and environmental factors contributed to individual differences in adolescent sleep duration, and whether genetic and environmental contributions to sleep duration changed throughout adolescence. A twin-family design was used to gain insight into the genetic and environmental contributions to variation in sleep duration. The study sample consisted of 6,319 adolescent twins ( $44 \%$ males) and 1,359 non-twin siblings ( $44 \%$ males) in the age range of 12 to 20 years (mean age = $16.85, S D=1.40$ ). The participants self-reported usual sleep duration, which was categorized as less than 8 hours per night, 8-9 hours per night, and more than 9 hours per night. Results showed that the prevalence of shorter than optimum sleep duration, that is, less than 8 hours per night, was high, with the highest prevalence rates in later adolescence. The contribution of genetic and environmental factors to individual differences in sleep duration was dependent on age. Variation in sleep duration at the age of 12 years was accounted for by genetic (boys: $34 \%$, girls: $36 \%$ ), shared environmental (boys: $28 \%$, girls: $45 \%$ ), and non-shared environmental factors (boys: $38 \%$, girls: 19\%). At the age of 20 years, the role of genetic (boys: 47\%, girls: $33 \%$ ) and non-shared environmental factors (boys: $53 \%$, girls: $67 \%$ ) was more pronounced. It can be concluded from the results that individual differences in sleep duration were accounted for by genetic and non-shared environmental factors throughout adolescence, whereas shared environmental factors account for a substantial part of variation during early adolescence only.
\end{abstract}

Keywords: sleep duration, adolescence, genetic epidemiology, twin-family study, sex differences

Sleep is essential in human life, and we spend about onethird of our lives sleeping. During childhood and adolescence, sleep plays an important role in growth, maturation, and physical and mental health (Mindell et al., 1999). Research regarding adolescents' sleep duration indicated that those reporting short sleep duration reported higher levels of depressed moods (Fallone et al., 2002; Moore \& Meltzer, 2008) and suboptimal performance at school (Fallone et al., 2002; Wolfson \& Carskadon, 2003) than those reporting optimal sleep duration. Sleep duration is also associated with physiological factors, such as glucose tolerance, insulin sensitivity, leptin and ghrelin levels, hunger, endocrine function, and appetite (Spiegel et al., 1999; Spruyt et al., 2011; Van Cauter \& Knutson, 2008; Van Cauter et al., 2008). In ad- dition, reviews and original studies have reported that short sleep duration is associated with overweight (Altenburg et al., 2013; Liu et al., 2012; Taheri et al., 2004); however, the evidence remains inconclusive due to methodological limitations (Guidolin \& Gradisar, 2012).

RECEIVED 19 September 2013; ACCEPTED 25 September 2013. First published online 4 November 2013.

ADDRESS FOR CORRESPONDENCE: Dr Meike Bartels, Department of Biological Psychology and $\mathrm{EMGO}^{+}$Institute for Health and Care Research, VU University, Van der Boechorststraat 5, 1081 BT Amsterdam, The Netherlands. E-mail: m.bartels@vu.nl *These authors contributed equally to the work. 
Despite the beneficial effects of adequate sleep, over past years a decline in sleep duration among children and adolescents has been observed (Matricciani et al., 2012), suggesting that many children and adolescents do not get adequate sleep. Moreover, studying sleep duration among adolescents is of particular interest because sleep duration on school nights steadily decreases from an average of 10 to 11 hours during childhood to approximately 8 hours during adolescence (Fallone et al., 2002; Iglowstein et al., 2003), that is, to sleep duration that is shorter than the amount of sleep they actually need during school nights (Carskadon \& Acebo, 2002; Carskadon et al., 1980). Adolescents start to feel sleepy later in the evening as a result of changes in the relative phase of circadian rhythms occurring during puberty (Carskadon \& Acebo, 2002; Moore \& Meltzer, 2008). In addition, for many adolescents' bedtimes are delayed due to, for example, increased amount of time spent on extracurricular activities (e.g., sports and music), jobs, homework, personal computer and Internet activities, playing video games, and social interaction with peers (Moore \& Meltzer, 2008). In combination with early school hours, late bedtimes result in shorter sleep duration (Hysing et al., 2013). The proportion of slow wave sleep (i.e., the restorative state of sleep) relative to rapid eye movement (REM) sleep decreases by approximately $40 \%$ during adolescence (Mindell et al., 1999; Moore \& Meltzer, 2008). To get the same amount of restorative sleep, adolescents' sleep duration should therefore be longer rather than shorter as compared with younger children's sleep (Carskadon \& Acebo, 2002; Carskadon et al., 1980). Adolescent sleep duration may therefore be a possible risk factor for suboptimal performance at school and dysregulation of physical and mental health.

To inform potential prevention and intervention strategies regarding adolescent sleep behavior, insight into the etiology of adolescent sleep duration is needed. Twin-family studies offer the possibility to gain insight into the sources of individual differences in sleep duration by disentangling the total variation into genetic variation, environmental variation due to influences shared by members of the same family (shared environmental influences), and environmental variation due to influences unique to an individual (nonshared environmental influences). A number of twin studies among adults investigated the relative influence of genetic and environmental factors on self-reported sleep duration, and typically reported individual differences in sleep duration were accounted for $30-40 \%$ by genetic factors, whereas the remaining part was accounted for by non-shared environmental factors (Barclay \& Gregory, 2013; de Castro, 2002; Gregory et al., 2006; Heath et al., 1990; Hublin et al., 2013; Partinen et al., 1983; Watson et al., 2010).

It is well known that adolescence is a period of important social, neurobiological, and behavioral changes (Paus et al., 2008). As a consequence, the etiology of sleep duration might change throughout adolescence. For example, influence of parents on adolescents' sleep duration is likely to decrease during adolescence, whereas the relative influence of extracurricular and social activities might increase. The relative contribution of genetic factors influencing biological processes regulating sleep, such as the circadian rhythm, may also increase during puberty. However, to our knowledge, it has not been investigated whether the relative contribution of genetic and environmental influences to individual differences in sleep duration is subject to change during adolescence.

In the present study, we assessed regular sleep duration in a large sample of Dutch adolescent twins and their nontwin siblings in the age range of 12 to 20 years. The main objective was to assess the contribution of genetic and environmental factors to individual differences in self-reported sleep duration during adolescence and whether the contribution of these factors was dependent on age.

\section{Methods}

\section{Participants}

Participants were registered as newborns with the Netherlands Twin Registry (NTR), which was established by the Department of Biological Psychology at the VU University in Amsterdam. In 2005, the NTR started to collect data on behavior, well-being, lifestyle, and health in adolescent twins and their non-twin siblings (Bartels et al., 2007; Boomsma et al., 2006, Van Beijsterveldt et al., 2013). During adolescence, 14-, 16-, and 18-year-old twins and their non-twin siblings (aged 12 to 25 years) received an online or a paper and pencil self-report survey upon written parental consent. A detailed description of the data collection procedures among adolescent twins registered with the NTR was reported elsewhere (Bartels et al., 2011). Data collection procedures and the content of self-report survey were approved by an institutional review board on human research.

For the present study, data from surveys collected in adolescent twins (born in cohorts 1986-1994) and their non-twin siblings were analyzed. Data from one additional non-twin sibling per family were included for the present study. From families with more than one additional participating sibling, we selected the sibling closest in age to the twin for the analyses. The total sample consisted of 6,319 twins (44\% male) and 1,359 non-twin siblings (44\% male) from 3,514 families. In Table 1, the exact constellation of the participating families is presented. Age of the twins and siblings ranged between 12 and 20 years. Mean age of the twins was 15.7 years $(S D=1.4)$. Mean age of the siblings was 16.9 years $(S D=2.3)$. Zygosity for $799(34.4 \%)$ of the same-sex twin pairs was determined on the basis of blood group or DNA typing. Zygosity for the remaining same-sex twin pairs was determined by questionnaire items about physical similarities and confusion obtained from family members and strangers, which were provided by parents 
TABLE 1

Sample Constellation

\begin{tabular}{|c|c|c|c|c|c|c|c|}
\hline & Number of individuals $(N)$ & Number of families $(N)$ & 1 twin & 2 twins & 0 twins + sibling & 1 twin + sibling & 2 twins + siblings \\
\hline MZM & 1,222 & 528 & 38 & 278 & - & 8 & 204 \\
\hline DZM & 1,031 & 455 & 47 & 224 & - & 16 & 168 \\
\hline MZF & 1,778 & 778 & 57 & 425 & - & 17 & 279 \\
\hline DZF & 1,250 & 559 & 61 & 295 & - & 10 & 193 \\
\hline DOS & 2,289 & 1,086 & 197 & 533 & - & 42 & 414 \\
\hline Siblings only & 108 & 108 & - & - & 108 & - & - \\
\hline Total & 7,678 & 3,514 & 400 & 1,755 & 108 & 93 & 1,158 \\
\hline
\end{tabular}

Note: 1 twin = families with data from one twin (incomplete twin pair); 2 twins = families with data from complete twin pairs; 0 twins + sibling $=$ families with data from one sibling; 1 twin + sibling = families with data from one twin (incomplete twin pair) and one sibling; 2 twins + siblings $=$ families with data from a complete twin pair and one additional sibling; $M Z=$ monozygotic twin pair; $D Z=$ dizygotic twin pair; $M=$ male; $F=$ female; Siblings only = families with data from non-twin siblings only.

on multiple time points in previous questionnaires. These items allow accurate determination of zygosity in $93 \%$ of the same-sex twin pairs (Rietveld et al., 2000).

\section{Measures}

The majority of participants (i.e., $83 \%$ ) were asked to indicate their usual sleep duration during a regular school or working week on a 3-point scale: $1=$ 'less than 8 hours per night' $(24.3 \%), 2=$ ' $8-9$ hours per night' $(66.8 \%)$, and $3=$ 'over 9 hours per night' (8.9\%). In the latest self-report surveys, the content of the item regarding sleep duration was slightly changed. Participants in the latest surveys (i.e., 17\% of the total sample) were asked to report on their usual sleep duration during the past four weeks on a 6-point scale: $1=$ ' 5 hours or less' $(0.7 \%), 2=$ ' 6 hours' $(4.4 \%), 3=$ ' 7 hours' $(17.4 \%), 4=$ ' 8 hours' (35.7\%), $5=$ ' 9 hours' (31.6\%), and $6=$ ' 10 hours or more' $(10.2 \%)$. For each response category the percentage of participants endorsed is shown in parentheses. It can be seen that the distributions of both items are similar. Therefore, data from both data collection procedures were combined by classifying the participants into three categories. The first category consisted of participants who indicated average sleep duration of less than 8 hours per night. The second category consisted of participants who reported average sleep duration between 8 and 9 hours. The third category consisted of those who indicated average sleep duration of over 9 hours per night. Although the self-report measure of sleep duration used in the present study was not formally validated, this measure has already been used in other twin-family studies assessing sleep duration (Heath et al., 1990; Partinen et al., 1983). It has been reported that among healthy individuals, measurement of sleep duration based on self-report is in agreement with objective laboratory-based measures of sleep duration based on polysomnography (Hoch et al., 1987; Manconi et al., 2010), indicating that self-report is a valid measure of sleep duration.

\section{Statistical Analyses}

In the following section, the statistical methods are briefly described. A more elaborate description is presented in the online supplement. (To view supplementary material for this article, please visit http://dx.doi.org/10.1017/thg.2013.74) Resemblance between twins and non-twin siblings is used to decompose individual differences in sleep duration as due to additive genetic (A), shared or common environmental (C), and non-shared environmental (E) factors (ACE model). Monozygotic (MZ) twin pairs are genetically identical, whereas dizygotic (DZ) twin pairs and twin-sibling pairs share on average $50 \%$ of their segregating genes. When the $M Z$ resemblance (expressed as a correlation) is higher than the resemblance in $\mathrm{DZ}$ twin or twin-sibling pairs, genetic variation is likely to influence individual differences in sleep duration. When MZ twin pairs resemble each other more than DZ twin and twin-sibling pairs, but not to the extent that would be expected based on their twice larger genetic resemblance, this implies that shared environmental factors influence variation in sleep duration. Other variation is attributed to non-shared environment. This component also includes measurement error variance.

As the data on sleep duration were ordinal, a liability threshold model was used. The basic assumption underlying the liability threshold model is that many genetic and environmental factors contribute to the liability of a trait (Falconer \& Mackay, 1996). The latent liability continuum is assumed to be a standard normal distribution $(S D=1)$. To model the three categories of sleep duration, two thresholds were specified that divide the sample into three categories. Twin and twin-sibling correlations were estimated on the liability scale for each of the five sex by zygosity groups (i.e., monozygotic male: MZM; dizygotic male: DZM; monozygotic female: MZF; dizygotic female: DZF; and dizygotic opposite-sex: DOS). In order to assess sex differences in prevalence of sleep duration and whether the prevalence of sleep duration changed with age, thresholds were estimated conditionally on sex and age before genetic modeling was started.

Next, genetic structural equation modeling in the software package Mx (Neale et al., 2006) was carried out to estimate the contribution of genetic, shared environmental, and non-shared environmental factors to individual 


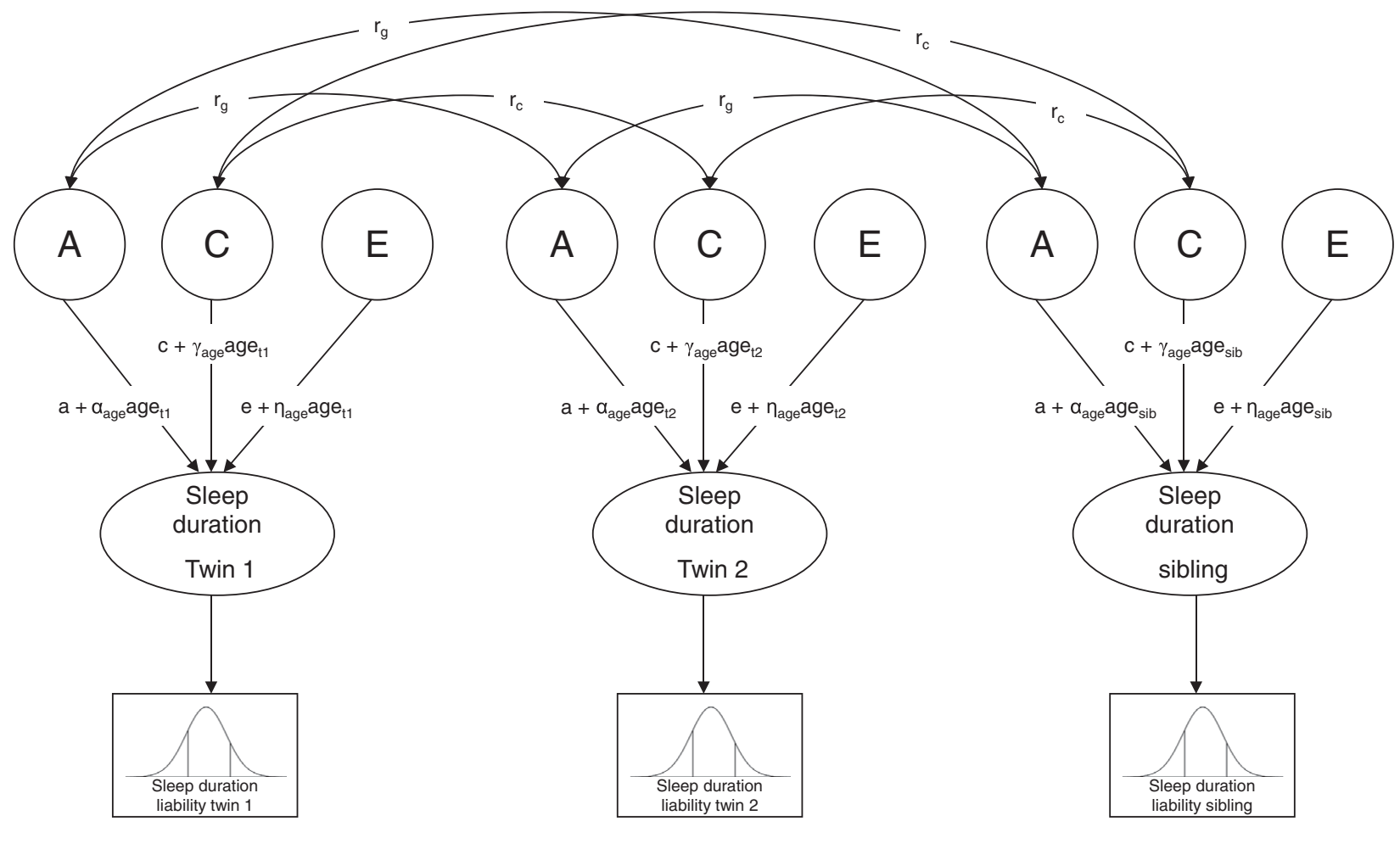

FIGURE 1

Univariate liability threshold model for sleep duration. Note: Sleep duration was measured with three categories. Thresholds were constrained at fixed values (i.e., 0 and 1) to enable the estimation of absolute variance of liability distribution. Total variance in sleep duration is modeled as caused by latent factors A (additive genetic influences), $C$ (shared environmental influences), and $E$ (non-shared environmental influences). Under this model, $a, c$, and e represent the unmoderated path coefficients, and $\alpha-\gamma_{-}$, and $\eta$-coefficients represent the moderating effects of age. If, for example, $\alpha$ is significantly different from zero, the magnitude of $A$ changes as a linear function of age. Path coefficients $a, c$, and $e$, as well as $\alpha-, \gamma-$, and $\eta$-coefficients, were allowed to differ for boys and girls. Genetic correlation $\left(r_{g}\right)$ : MZ twin pairs = 1, DZ twin pairs and twin-sibling pairs = 0.5; shared environmental correlation $\left(r_{c}\right)=1$.

differences in liability (Figure 1). A moderator model as described by Purcell (2002) was fitted to the data in which age ( $z$-score) was regressed on the genetic $(a)$, shared environmental $(c)$, and non-shared environmental (e) path coefficients (for details see supplementary material online). In Figure 1, regression weights of age are represented by the $\alpha$, $\gamma$, and $\eta$ coefficients. As described by Medland et al. (2009), in the moderator model thresholds were constrained at fixed values (i.e., 0 and 1) to enable the estimation of the variance of the latent liability distribution of sleep duration, and hence the absolute contribution of genetic and environmental factors to the variance. To assess sex differences in the genetic architecture of sleep duration, the regression coefficients of age as well as the path coefficients, $a, c$, and $e$, were allowed to differ for boys and girls, and it was tested whether constraining these parameter estimates to be equal for boys and girls reduced model fit significantly. The significance of the age effects was also assessed by testing whether constraining the regression weights of age at zero resulted in a significant deterioration of model fit. Finally, to assess whether genetic and shared environmental factors contributed significantly to individual differences in liability, we tested whether constraining the parameter estimates for $\mathrm{A}$ and $\mathrm{C}$ (i.e., $a$ and $c$ ) at zero resulted in a significant deterioration of model fit.

Submodels were nested in the sense that one model could be derived from the other by the imposition of one or more constraints on the parameters. The fit of submodels was compared by means of the log-likelihood ratio test (LRT). If a $p$ value higher than .05 was obtained from the resulting $\chi^{2}$-test statistic, the fit of the constrained model was not significantly worse than the fit of the more complex model. The fit of the genetic models was also compared with the full ACE model by means of Akaike's Information Criterion (AIC), keeping the model with the lowest AIC as the best fitting model (Neale et al., 2006).

\section{Results}

Figure 2 presents the prevalence of sleep duration as a function of age and sex. For boys and girls, short sleep duration ( $<8$ hours) was more prevalent among older adolescents, whereas long ( $>9$ hours) and average sleep durations (8-9 hours) were more prevalent among younger adolescents. These age differences in the prevalence of sleep duration were larger for boys than girls. Formal tests on the 
Boys

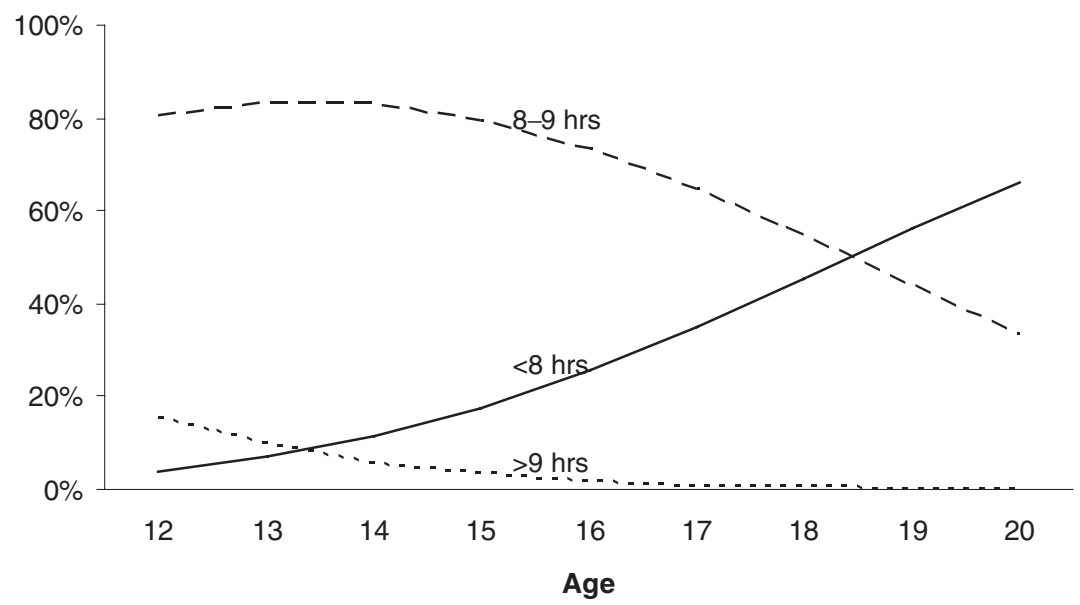

\section{Girls}

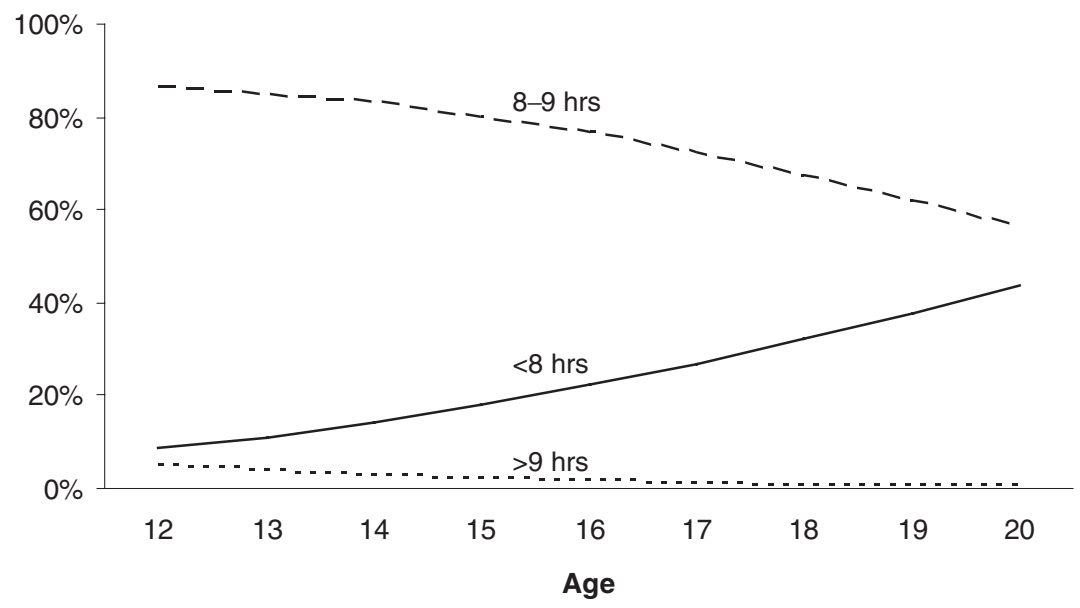

\section{FIGURE 2}

Changes in the prevalence of sleep duration as a function of sex and age.

thresholds showed these age effects on the prevalence of sleep duration and sex differences in the magnitude of these age effects to be significant. The magnitude of age effects on the first, $\chi^{2}(1)=25.02, p<.01$, and the second threshold, $\chi^{2}(1)=23.29, p<.01$, was significantly different for boys and girls. Age effects on the thresholds were significant for boys: first threshold: $\chi^{2}(1)=259.70, p<.01$, standardized regression coefficient $\beta=0.45$; second threshold: $\chi^{2}(1)=$ $154.18, p<.01$, standardized regression coefficient $\beta=0.48$; and for girls: first threshold: $\chi^{2}(1)=117.81, p<.01$, standardized regression coefficient $\beta=0.25$; second threshold: $\chi^{2}(1)=58.15, p<.01$, standardized regression coefficient $\beta=0.22$.

Twin and twin-sibling correlations for sleep duration, uncorrected for age, are presented in Table 2. DZ twin correlations were not significantly different from twin-sibling correlations, $\chi^{2}(3)=5.70, p=.13$. The MZ twin correlations were significantly higher than the DZ twin or twin-sibling correlations, boys: $\chi^{2}(1)=24.94, p<.01$, girls: $\chi^{2}(1)=$ $39.82, p<.01$. This suggests that individual differences in sleep duration are influenced by genetic factors.

The genetic model fitting revealed that there were no sex differences in the magnitude of age effect on the genetic, $(\alpha) p=.80$, and shared environmental, $(\gamma) p=.67$, path coefficients, whereas the magnitude of the age effect on the non-shared environmental $(\eta)$ path coefficient was significantly different between boys and girls, $p \leq .05$. The age effects on the shared environmental $(\gamma)$ and non-shared environmental path coefficient for girls $\left(\eta_{\text {girls }}\right)$ were statistically significant, $p<.05$. This indicates that the contribution of shared (for boys and girls) and non-shared environmental factors (for girls only) to variation in sleep duration 
Boys: Unstandardized variance components

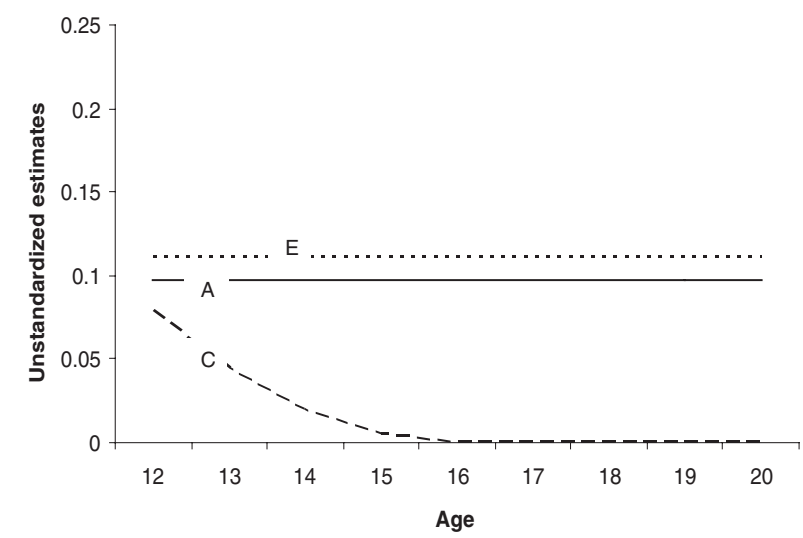

Girls: Unstandardized variance components

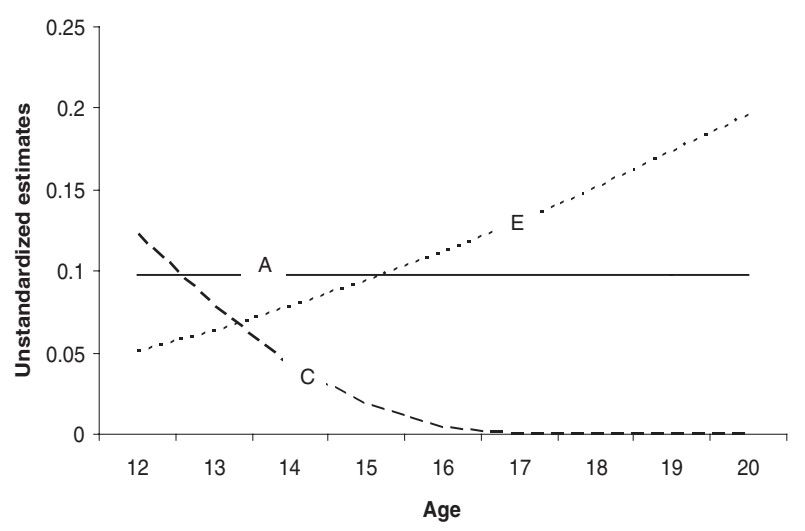

Boys: Standardized variance components

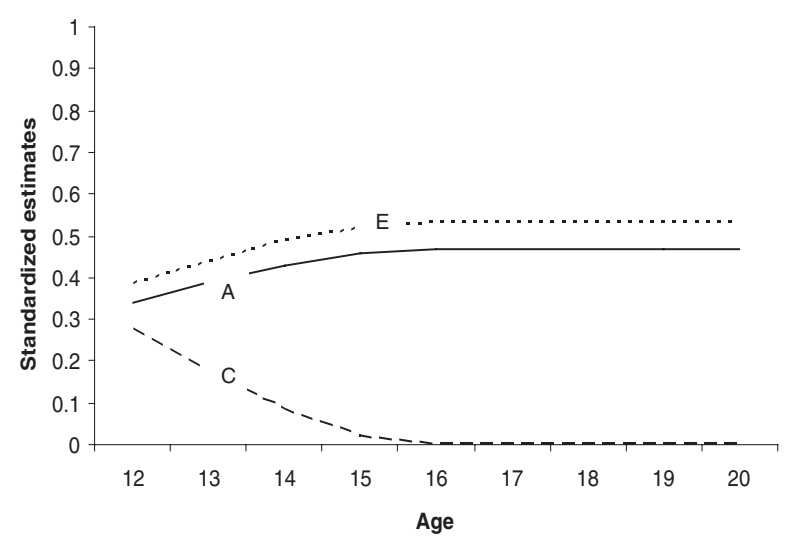

Girls: Standardized variance components

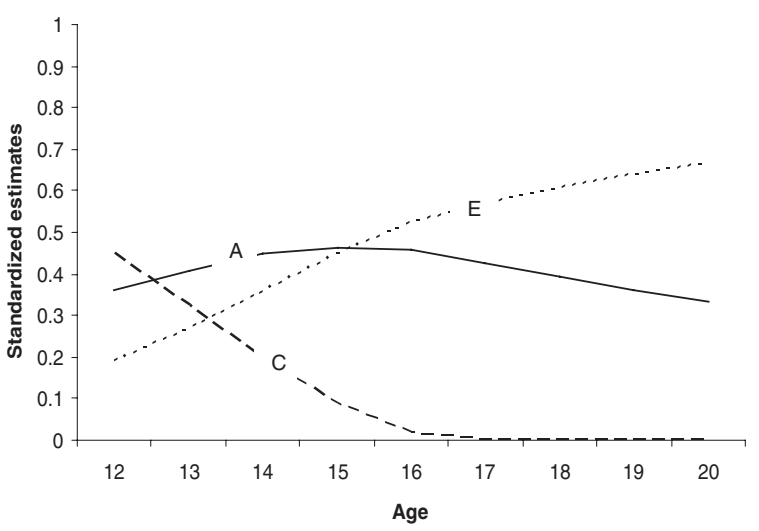

FIGURE 3

Changes in the absolute and relative contributions of genetic, shared environmental, and non-shared environmental effects to variation in sleep duration as a function of age for boys and girls.

\section{TABLE 2}

Polychoric Twin and Twin-Sibling Correlations, Uncorrected for Age*, and Their 95\% Confidence Intervals (Cl) for Sleep Duration

\begin{tabular}{lll}
\hline & $r$ & $95 \% \mathrm{Cl}$ \\
\hline MZM & 0.54 & $0.47-0.61$ \\
DZM & 0.28 & $0.18-0.38$ \\
MZF & 0.56 & $0.50-0.62$ \\
DZF & 0.25 & $0.15-0.35$ \\
DOS & 0.19 & $0.12-0.26$ \\
Brother & 0.08 & $0.00-0.16$ \\
Sister & 0.17 & $0.11-0.23$ \\
Brother-sister & 0.16 & $0.06-0.26$
\end{tabular}

Note: Brother = male twin-brother pairs; Sister = female twin-sister pairs; Brother-sister = male twin-sister pairs, female twin-brother pairs. *Results in twin correlations for the mean age of the sample $(=15.9)$.

changed as a function of age. The age effects on the genetic $(\alpha)$ and non-shared environmental path coefficient for boys ( $\eta_{\text {boys }}$ ) were not statistically significant. The absolute and relative changes in contribution of genetic, shared environ- mental, and non-shared environmental factors to variation in sleep duration as a function of age and sex can be seen in Figure 3.

Next we tested whether constraining the genetic, shared environmental, and non-shared environmental parameter estimates to be equal for boys and girls led to a significant deterioration of model fit. There appeared to be significant sex differences in the magnitude of the shared environmental path coefficient, while the magnitude of the genetic and non-shared environmental path coefficients was similar for boys and girls. Shared environmental effects on individual differences in sleep duration were statistically significant for girls but not for boys. Note, however, that the age effect on shared environment is significant for both boys and girls, resulting in the presence of shared environmental influences at the beginning of adolescence for both boys and girls (see Figure 3). Additive genetic effects on variation in sleep duration were statistically significant. Statistical analyses (LRT tests and the AIC, see supplementary material online) pointed to a 
model in which variance in sleep duration was explained by genetic, shared environmental, and non-shared environmental factors with significant age effects on the shared environmental path coefficients for boys and girls and the non-shared environmental path coefficient for girls as the most parsimonious model. By constraining the thresholds to fixed values, the variance of the latent liability distribution of sleep duration could be estimated and, hence the absolute magnitude of genetic, shared environmental, and non-shared environmental effect on the variance of sleep duration (Medland et al., 2009).

Figure 3 presents the unstandardized (presented on the left side) and standardized (presented on the right side) contribution of genetic, shared environmental, and nonshared environmental factors to variation in sleep duration as a function of age and sex, derived from the most parsimonious model. For boys and girls, shared environmental factors contributed to variation in sleep duration among younger, but not among older adolescents. For boys, the absolute contribution of genetic and non-shared environmental factors to variation in sleep duration was similar for all ages, whereas the relative contribution of genetic and non-shared environmental effects was smaller in younger compared with older participants. This is due to shared environmental influences on variation in sleep duration for younger boys and the absence of these influences among older boys, leading to an overall lower variation in sleep duration among older boys. For girls, the absolute and relative contribution of non-shared environmental effects to variation in sleep duration was larger in older than younger participants. The absolute contribution of genetic effects to variation in sleep duration was similar at all ages, whereas the relative contribution of genetic effects was larger in younger compared with older girls. This is due to the fact that the contribution of shared and nonshared environmental factors to variation in sleep duration was different for younger and older girls, leading to differences in absolute variation in sleep duration throughout adolescence.

\section{Discussion}

In a large sample of Dutch adolescent twins and their siblings, shorter than optimum sleep duration, that is, less than 8 hours per night, was more prevalent among older compared to younger adolescents. This is in line with previous studies that reported sleep duration became shorter during adolescence (Fallone et al., 2002; Iglowstein et al., 2003). Variation in sleep duration was accounted for by genetic, shared environmental, and non-shared environmental factors. In addition, the contribution of shared and non-shared environmental influences to individual differences in sleep duration differed by age. Specifically, shared environmental effects on sleep duration were important during early adolescence, but were of no importance during middle and late adolescence. For girls, non-shared environmental effects on sleep duration were larger among older than younger adolescents. For boys and girls, the absolute contribution of genetic factors to individual differences in sleep duration was similar at all ages, as was the case for non-shared environmental factors for boys.

The present study showed individual differences in adolescent sleep duration to be accounted for $35-45 \%$ by genetic factors. The absolute influence of genetic factors on individual differences in sleep duration was similar for all ages. Genetic factors on sleep duration may influence biological processes such as the biological circadian timing system, which, in turn, affects sleep duration. Changes in the relative phase of circadian rhythm, a process occurring during adolescence, cause adolescents to feel sleepy later in the evening (Carskadon \& Acebo, 2002; Moore \& Meltzer, 2008). A number of genetic variants, such as the Per3, CLOCK, TRIB1, Cry1, and Cry2 genes, have been associated with circadian rhythmicity, slow wave sleep, and abnormalities in the circadian timing system (Cirelli, 2009; Dauviltiers et al., 2005; Ollila et al., 2012; Tafti, 2009; Wulff et al., 2009) and some have also been linked to depression and lipid metabolism (Ollila et al., 2012; Utge et al., 2010).

Due to a genetically defined 'preference' for late bedtimes (Carskadon \& Acebo, 2002; Moore \& Meltzer, 2008), increasing adolescents' sleep duration by advancing bed times may not be feasible for many middle and late adolescents. Instead, delaying the onset of school days may have better perspective for these adolescents (Kirby et al., 2011). Previous studies have reported that when high school start times are delayed, adolescents' bedtimes do not change and, consequently, sleep duration increases (Owens et al., 2010; Wahlstrom, 2002; Wolfson et al., 2007). It must be noted that lack of sleep is not suffered by the entire adolescent population because of a preference for late bedtimes. It has been reported that the prevalence of suboptimal sleep is significantly smaller among adolescents with a preference for early bed and rising times than those with a preference for late bedtimes (Giannotti et al., 2002).

For early adolescents, shared environmental factors accounted for a major part of individual differences in sleep duration, whereas the contribution of shared environmental was absent among middle and late adolescents. Shared environmental factors may include the influence of parents on early adolescents' sleep duration by setting rules about bed and wakening times. It has been reported that parents set fewer rules about bedtimes throughout adolescence (Carskadon, 1990, 2002), which is in line with the finding of the present study that the influence of shared environmental factors on sleep duration is larger for early adolescents compared with middle and late adolescents. The diminishing contribution of shared environmental factors during early adolescence is in line with other phenotypes that have 
been assessed among adolescents, such as exercise behavior (Van der Aa et al., 2010), sedentary behavior (Van der Aa et al., 2012), and psychopathology (Bartels et al., 2011; Lamb et al., 2010).

For early adolescent girls, non-shared environmental factors accounted for a modest part of variation in sleep duration, whereas non-shared environmental factors accounted for a major part of individual differences in sleep duration among middle and late adolescent girls. For boys, nonshared environmental factors constituted the main source of variation in sleep duration throughout adolescence and the contribution of these factors was similar for all ages. Factors from adolescents' personal environment that have previously been associated with individual differences in bedtime and hence sleep duration include participation in sports late in the evening, after-school employment, homework, engagement in personal computer and internet activities, playing video games, and increasing amount of time in the evening spent in social interaction with peers (Moore \& Meltzer, 2008).

Additional analyses in our own data indicated that sleep duration is associated with time spent in playing video games, engagement in personal computer and internet activities, social interaction with peers, and after-school employment. Specifically, the prevalence of suboptimal sleep duration (i.e., less than 8 hours per night) was approximately $10 \%$ higher among adolescents who reported to spend 3 hours or more on playing video games and engagement in personal computer and internet activities compared with those spending less time on these activities. Among adolescents engaged in after-school employment, prevalence of suboptimal sleep duration was about $10 \%$ higher than among those reporting no after-school employment. Prevalence of short sleep duration was also approximately $10 \%$ higher for adolescents spending time in social interaction with peers on a daily basis versus those reporting to be less frequently engaged in social interaction with peers. This indicates that changes in the social and economic environments that emerge during adolescence result in suboptimal sleep duration for some adolescents, whereas for others these changes do not affect their sleep duration. No indication was found, however, that sleep duration is associated with educational level, watching television, and participation in exercise. Prevalence of short, normal, and long sleep duration was similar for participants enrolled in lower, moderate, and higher secondary education, as well as for vigorous, moderate, and non-exercisers and those reporting to spend 3 hours or more and less than 3 hours on watching television. Although schooldays are longer for adolescents enrolled in higher levels of secondary education and they have more homework than those enrolled in lower levels of secondary education, no indication was found that this affects sleep duration. Exercise activities and TV shows aimed at adolescents are taking place later in the evening compared with those aimed at younger children, but no evidence was found that this negatively affects adolescent sleep duration.

An explanation for why the influence of non-shared environmental factors on individual differences in sleep duration is larger for boys than girls during early adolescence is that young adolescent boys already spend more time in activities that shorten their sleep duration by delayed bedtimes than girls. For example, adolescent boys are more frequently engaged than girls in sedentary activities, including engagement in personal computer and Internet activities and playing video games (Van der Aa et al., 2012). An alternative explanation is that girls are more obedient toward parental control over sleep duration than boys. Spilsbury et al. (2005) reported that a parenting style encouraging social maturity was associated with healthy sleep patterns among girls but not boys. Parental control over sleep duration may also be larger for early adolescent girls compared with boys, such that parental control over sleep duration may prevent early adolescent girls engaging in the activities associated with shortened sleep duration late in the evening. This might explain the modest influence of non-shared environmental factors on individual differences in sleep duration among young adolescent girls. Parents are more likely to set clear expectations for behavior to daughters than to sons (Radziszewska et al., 1996).

To our knowledge, the genetic architecture of adolescent sleep duration has not been addressed before, although some studies have investigated this among younger children (Brescianini et al., 2011; Gregory et al., 2006) or addressed sleep problems among youth (Barclay \& Gregory, 2013; Gehrman et al., 2011; Gregory et al., 2006). However, a number of studies assessed the relative influence of genetic and environmental factors on sleep duration among adults (de Castro, 2002; Heath et al., 1990; Hublin et al., 2013; Partinen et al., 1983; Watson et al., 2010). These studies showed that genetic factors accounted for $30-40 \%$ of individual differences in adult sleep duration, which is consistent with the findings of our study. The remaining part of variation in adult sleep duration is accounted for by non-shared environmental factors, which is also in line with our findings among middle and late adolescents.

A limitation to the present study was the use of a crosssectional twin-family design to assess whether the contribution of genetic and environmental factors to individual differences in sleep duration is subject to change during the different stages of adolescence. This would be most properly addressed in a longitudinal design. So far, data at two time points are only available for a small sub-sample, and longitudinal data throughout adolescence (12-20 years of age) are absent. As our data collection is a continuous process at the NTR, large enough longitudinal sample sizes are anticipated in the next 5 years. 


\section{Conclusions}

Our data showed that individual differences in adolescent sleep duration were accounted for by genetic and nonshared environmental factors, whereas shared environmental factors accounted for a substantial part of variation among early adolescents. For girls, the contribution of nonshared environmental factors to individual differences in sleep duration was larger among older adolescents compared with younger adolescents, while for boys, non-shared environmental factors constituted the main source of individual differences in sleep duration throughout adolescence and the contribution of these factors was similar at all ages.

\section{Acknowledgments}

Funding was obtained from the following grants: 'Genes and environment in obesogenic behaviour: Exploring the relative importance of environmental and genetic factors on sedentary behaviour and sleep duration during adolescence - a study in adolescent twins' (WCRF 2009/08); 'Twinfamily database for behavior genetics and genomics studies' (NWO 480-04-004); 'Spinozapremie' (NWO/SPI 56-46414192); 'Genetic and family influences on adolescent psychopathology and wellness' (NWO 463-06-001); 'A twinsibling study of adolescent wellness' (NWO-VENI 451-04034); 'Determinants of adolescent exercise behavior; towards evidence-based intervention' (National Institute of Diabetes and Digestive and Kidney Diseases [NIDDK], RO1DK092127).

\section{References}

Altenburg, T. M., Chinapaw, M. J., van der Knaap, E. T., Brug, J., Manios, Y., \& Singh, A. S. (2013). Longer sleep - Slimmer kids: The ENERGY-project. PLoS One, 8, e59522.

Barclay, N. L., \& Gregory, A. M. (2013). Quantitative genetic research on sleep: A review of normal sleep, sleep disturbances and associated emotional, behavioural, and healthrelated difficulties. Sleep Medicine Reviews, 17, 29-40.

Bartels, M., van Beijsterveldt, C. E. M., Derks, E. M., Stroet, T. M., Polderman, T. J. C., Hudziak, J. J., \& Boomsma, D. I. (2007). Young Netherlands Twin Register (Y-NTR): A longitudinal multiple informant study of problem behavior. Twin Research and Human Genetics, 10, 3-11.

Bartels, M., Van der Aa, N., van Beijsterveldt, C. E. M., Middeldorp, C. M., \& Boomsma, D. I. (2011). Adolescent self-report of emotional and behavioral problems: Interactions of genetic factors with sex and age. Journal of the Canadian Acadademy of Child and Adolescent Psychiatry, 20,35-52.

Boomsma, D. I., de Geus, E. J. C., Vink, J. M., Stubbe, J. H., Distel, M. A., Hottenga, J. J., . . Willemsen, G. (2006). Netherlands twin register: From twins to twin families. Twin Research and Human Genetics, 9, 849-857.

Brescianini, S., Volzone, A., Fagnani, C., Patriarca, V., Grimaldi, V., Lanni, R., . . . Stazi, M. A. (2011). Genetic and environmental factors shape infant sleep patterns: A study of 18-month-old twins. Pediatrics, 127, 1296-1302.

Carskadon, M. A. (1990). Patterns of sleep and sleepiness in adolescents. Pediatrician, 17, 5-12.

Carskadon, M. A. (2002). Factors influencing sleep patterns of adolescents. In M. A. Carskadon (Ed.), Adolescent sleep patterns: Biological, social, and psychological influences (pp. 4-26). New York, NY: Cambridge University Press.

Carskadon, M. A., \& Acebo, C. (2002). Regulation of sleepiness in adolescents: Update, insights, and speculation. Sleep, 25, 606-614.

Carskadon, M. A., Harvey, K., Duke, P., Anders, T. F., Litt, I. F., \& Dement, W. C. (1980). Pubertal changes in daytime sleepiness. Sleep, 2, 453-460.

Cirelli, C. (2009). The genetic and molecular regulation of sleep: From fruit flies to humans. Nature Reviews Neuroscience, 10, 549-560.

Dauviltiers, Y., Maret, S., \& Tafti, M. (2005). Genetics of normal and pathological sleep in humans. Sleep Medicine Reviews, 9, 91-100.

de Castro, J. M. (2002). The influence of heredity on selfreported sleep patterns in free-living humans. Physiology and Behavior, 76, 479-486.

Falconer, D. S., \& Mackay, T. F. C. (1996). Introduction to quantitative gentics. Essex, UK: Pearson.

Fallone, G., Owens, J. A., \& Deane, J. (2002). Sleepiness in children and adolescents: Clinical implications. Sleep Medicine Reviews, 6, 287-306.

Gehrman, P. R., Meltzer, L. J., Moore, M., Pack, A. I., Perlis, M. L., Eaves, L. J., \& Silberg, J. L. (2011). Heritability of insomnia symptoms in youth and their relationship to depression and anxiety. Sleep, 34, 1641-1646.

Giannotti, F., Cortesi, F., Sebastiani, T., \& Ottaviano, S. (2002). Circadian preference, sleep and daytime behaviour in adolescence. Journal of Sleep Research, 11, 191-199.

Gregory, A. M., Rijsdijk, F. V., \& Eley, T. C. (2006). A twinstudy of sleep difficulties in school-aged children. Child Development, 77, 1668-1679.

Guidolin, M., \& Gradisar, M. (2012). Is shortened sleep duration a risk factor for overweight and obesity during adolescence? A review of the empirical literature. Sleep Medicine, 13, 779-786.

Heath, A. C., Kendler, K. S., Eaves, L. J., \& Martin, N. G. (1990). Evidence for genetic influences on sleep disturbance and sleep pattern in twins. Sleep, 13, 318-335.

Hoch, C. C., Reynolds, C. F., Kupfer, D. J., Berman, S. R., Houck, P. R., \& Stack, J. A. (1987). Empirical note - Selfreport versus recorded sleep in healthy seniors. Psychophysiology, 24, 293-299.

Hublin, C., Partinen, M., Koskenvuo, M., \& Kaprio, J. (2013). Genetic factors in evolution of sleep length - A longitudinal twin study in Finnish adults. Journal of Sleep Research, 22, 513-518.

Hysing, M., Pallesen, S., Stormark, K. M., Lundervold, A. J., $\&$ Sivertsen, B. (2013). Sleep patterns and insomnia among adolescents: A population-based study. Journal of Sleep Research, 22, 549-556. 
Iglowstein, I., Jenni, O. G., Molinari, L., \& Largo, R. H. (2003). Sleep duration from infancy to adolescence: Reference values and generational trends. Pediatrics, 111, 302307.

Kirby, M., Maggi, S., \& D’Angiulli, A. (2011). School start times and the sleep-wake cycle of adolescents: A review and critical evaluation of available evidence. Educational Researcher, 40, 56-61.

Lamb, D. J., Middeldorp, C. M., van Beijsterveldt, C. E. M., Bartels, M., Van der Aa, N., Polderman, T. J. C., \& Boomsma, D. I. (2010). Heritability of anxious-depressive and withdrawn behavior: Age-related changes during adolescence. Journal of the American Academy of Child and Adolescent Psychiatry, 49, 248-255.

Liu, J., Zhang, A., \& Li, L. (2012). Sleep duration and overweight/obesity in children: Review and implications for pediatric nursing. Journal for Specialists in Pediatric Nursing, 17, 193-204.

Manconi, M., Ferri, R., Sagrada, C., Punjabi, N. M., Tettamanzi, E., Zucconi, M., . . Ferini-Strambi, L. (2010). Measuring the error in sleep estimation in normal subjects and in patients with insomnia. Journal of Sleep Research, 19, 478-486.

Matricciani, L., Olds, T., \& Petkov, J. (2012). In search of lost sleep: Secular trends in the sleep time of school-aged children and adolescents. Sleep Medicine Reviews, 16, 203211.

Medland, S. E., Neale, M. C., Eaves, L. J., \& Neale, B. M. (2009). A note on the parameterization of Purcell's $\mathrm{G} \times \mathrm{E}$ model for ordinal and binary data. Behavior Genetics, 39, 220-229.

Mindell, J. A., Owens, J. A., \& Carskadon, M. A. (1999). Developmental features of sleep. Child and Adolescent Psychiatric Clinics of North America, 8, 695-725.

Moore, M., \& Meltzer, L. J. (2008). The sleepy adolescent: Causes and consequences of sleepiness in teens. Paediatric Respiratory Reviews, 9, 114-121.

Neale, M. C., Boker, S. M., Xie, G., \& Maes, H. M. (2006). Mx: Statistical modeling (7th ed.). Richmond, VA: Department of Psychiatry.

Ollila, H. M., Utge, S., Kronholm, E., Aho, V., Van, L. W., Silander, K., . . . Paunio, T. (2012). TRIB1 constitutes a molecular link between regulation of sleep and lipid metabolism in humans. Translational Psychiatry, 2, e97.

Owens, J. A., Belon, K., \& Moss, P. (2010). Impact of delaying school start time on adolescent sleep, mood, and behavior. Archives of Pediatrics \& Adolescent Medicine, 164, 608-614.

Partinen, M., Kaprio, J., Koskenvuo, M., Putkonen, P., \& Langinvainio, H. (1983). Genetic and environmental determination of human sleep. Sleep, 6, 179-185.

Paus, T., Keshavan, M., \& Giedd, J. N. (2008). Why do many psychiatric disorders emerge during adolescence? Nature Reviews Neuroscience, 9, 947-957.

Purcell, S. (2002). Variance components models for geneenvironment interaction in twin analysis. Twin Research, 5, 554-571.

Radziszewska, B., Richardson, J. L., Dent, C. W., \& Flay, B. R. (1996). Parenting style and adolescent depressive symp- toms, smoking, and academic achievement: Ethnic, gender, and SES differences. Journal of Behavioral Medicine, 19, 289-305.

Rietveld, M. J., van Der Valk, J. C., Bongers, I. L., Stroet, T. M., Slagboom, P. E., \& Boomsma, D. I. (2000). Zygosity diagnosis in young twins by parental report. Twin Research, 3, 134-141.

Spiegel, K., Leproult, R., \& Van Cauter, E. (1999). Impact of sleep debt on metabolic and endocrine function. Lancet, 354, 1435-1439.

Spilsbury, J. C., Storfer-Isser, A., Drotar, D., Rosen, C. L., Kirchner, H. L., \& Redline, S. (2005). Effects of the home environment on school-aged children's sleep. Sleep, 28, 14191427.

Spruyt, K., Molfese, D. L., \& Gozal, D. (2011). Sleep duration, sleep regularity, body weight, and metabolic homeostasis in school-aged children. Pediatrics, 127, e345-e352.

Tafti, M. (2009). Genetic aspects of normal and disturbed sleep. Sleep Medicine, 10, S17-S21.

Taheri, S., Lin, L., Austin, D., Young, T., \& Mignot, E. (2004). Short sleep duration is associated with reduced leptin, elevated ghrelin, and increased body mass index. PLoS Medicine, 1, 210-217.

Utge, S. J., Soronen, P., Loukola, A., Kronholm, E., Ollila, H. M., Pirkola, S., . . Paunio, T. (2010). Systematic analysis of circadian genes in a population-based sample reveals association of TIMELESS with depression and sleep disturbance. PloS One, 5, e9259.

Van Beijsterveldt, C. E. M., Groen-Blokhuis, M. M., Hottenga, J. J., Franić, S., Hudziak, J. J., Lamb, D., . . Boomsma, D. I. (2013). The Young Netherlands Twin Register (YNTR): Longitudinal twin and family studies in over 70,000 children. Twin Research and Human Genetics, 16, 252267.

Van Cauter, E., \& Knutson, K. L. (2008). Sleep and the epidemic of obesity in children and adults. European Journal of Endocrinology, 159, S59-S66.

Van Cauter, E., Splegel, K., Tasali, E., \& Leproult, R. (2008). Metabolic consequences of sleep and sleep loss. Sleep Medicine, 9, S23-S28.

Van der Aa, N., Bartels, M., te Velde, S. J., Boomsma, D. I., de Geus, E. J., \& Brug, J. (2012). Genetic and environmental influences on individual differences in sedentary behavior during adolescence: A twin-family study. Archives of Pediatrics \& Adolescent Medicine, 166, 509-514.

Van der Aa, N., de Geus, E. J. C., van Beijsterveldt, T. C. E. M., Boomsma, D. I., \& Bartels, M. (2010). Genetic influences on individual differences in exercise behavior during adolescence. International Journal of Pediatrics, 138345.

Wahlstrom, K. (2002). Changing times: Findings from the first longitudinal study of later high school start times. NASSP Bulletin, 86, 3-21.

Watson, N. F., Buchwald, D., Vitiello, M. V., Noonan, C., \& Goldberg, J. (2010). A twin study of sleep duration and body mass index. Journal of Clinical Sleep Medicine, 6, 1117. 
Wolfson, A. R., \& Carskadon, M. A. (2003). Understanding adolescents' sleep patterns and school performance: A critical appraisal. Sleep Medicine Reviews, 7, 491506.

Wolfson, A. R., Spaulding, N. L., Dandrow, C., \& Baroni, E. M. (2007). Middle school start times: The importance of a good night's sleep for young adolescents. Behavioral Sleep Medicine, 5, 194-209.

Wulff, K., Porcheret, K., Cussans, E., \& Foster, R. G. (2009). Sleep and circadian rhythm disturbances: Multiple genes and multiple phenotypes. Current Opinion in Genetics \& Development, 19, 237-246. 